\title{
Immunotherapy in head and neck squamous cell carcinoma and rare head and neck malignancies
}

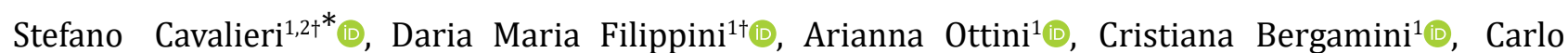

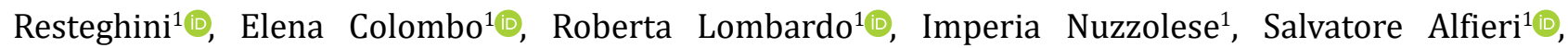
Lisa Licitra ${ }^{1,2}$, Laura D. Locati ${ }^{1}$ (])

${ }^{1}$ Head and Neck Cancer Medical Department, Fondazione IRCCS Istituto Nazionale dei Tumori di Milano, via Venezian 1, 20133 Milan, Italy

${ }^{2}$ Department of Oncology and Hemato-Oncology, University of Milan, via Festa del Perdono 7, 20122 Milan, Italy

${ }^{\dagger}$ These authors share the first authorship.

${ }^{*}$ Correspondence: Stefano Cavalieri, Head and Neck Cancer Medical Department, Fondazione IRCCS Istituto Nazionale dei Tumori di Milano, via Venezian 1, 20133 Milan, Italy; Department of Oncology and Hemato-Oncology, University of Milan, via Festa del Perdono 7, 20122 Milan, Italy. stefano.cavalieri@istitutotumori.mi.it

Academic Editor: Rossana Berardi, Università Politecnica Marche, Italy

Received: April 2, 2021 Accepted: October 29, 2021 Published: December 31, 2021

Cite this article: Cavalieri S, Filippini DM, Ottini A, Bergamini C, Resteghini C, Colombo E, et al. Immunotherapy in head and neck squamous cell carcinoma and rare head and neck malignancies. Explor Target Antitumor Ther. 2021;2:522-42. https:// doi.org/10.37349/etat.2021.00062

\begin{abstract}
The dismal prognosis of recurrent/metastatic (R/M) head and neck squamous cell carcinoma (HNSCC) prompted recent advances in the field of therapeutic approaches beyond cytotoxic cancer therapy. In recent years, the deeper and increasing knowledge on the genomic landscape and the upcoming new data on immunotherapy enacted by HNSCCs have led to successful therapeutic targeting of the immune system. Immune checkpoint inhibitors (ICIs) have changed state of the art in R/M patients and could have a potential role even in early disease. The purpose of this work is to summarize the role of immunotherapy for R/M HNSCC in clinical practice, with insights about future perspectives. Updated immunotherapy results in other R/M head and neck cancers such as thyroid, salivary glands, nasopharynx, sinonasal cancers, and nuclear protein in testis (NUT) are presented.
\end{abstract}

\section{Keywords}

Head and neck cancer, immune checkpoint inhibitors, rare cancer

\section{Introduction}

Head and neck cancers (HNC) are a heterogeneous group of malignancies, including several histotypes, and head and neck squamous cell carcinoma (HNSCC) is the most frequent. They may arise from the oral cavity, oropharynx, hypopharynx, larynx, and nasopharynx. This latter has a distinct clinical behavior and unique biology related to Epstein-Barr virus (EBV) infection. Another oncogenic virus involved in HNSCC etiopathogenesis is the human papillomavirus (HPV), almost correlated to oropharyngeal cancer (OPC). 
HPV-related HNSCCs have a distinct immune landscape compared to HPV-negative cases. The presence of diffuse immune infiltrates including $\mathrm{CD}^{+}$, Tregs, natural killer (NK) cells, B cells, and in HPV-related HNSCC suggest that checkpoint inhibitors may be beneficial for these patients $[1,2]$. By contrast, HPV-negative cancers have a lower number of programmed cell death protein 1 (PD-1) expressing $\mathrm{CD}^{+}$and $\mathrm{CD}^{+} \mathrm{T}$ cells than their HPV-positive counterparts [3]. Meanwhile, the existence of a high mutation burden in HPV-negative tumors, characterized by a high degree of smoking-related signatures, is a favorable opportunity for antitumor immunity to tackle the dominant immunosuppressive environment. However, the current results with checkpoint inhibitors in HPV-related cancers are still contradictory.

The landscape of immunotherapy in recurrent/metastatic (R/M) HNSCC has been rapidly evolving in the last years, and many clinical studies have been published so far. In the medical communities, the most famous ones are those that led to the regulatory approval of immune checkpoint inhibitors (ICIs) in clinical practice, but several other studies were negative, failing to provide a survival benefit in HNSCC patients. Moreover, the main achievements have been reached in the R/M setting, but more recently the research has been focusing on loco-regionally advanced potentially curable diseases.

In this review, we summarized the actual ICIs for R/M HNCs in clinical practice, outlining the results of the most recent clinical trials and looking to future perspectives. Even though the vast majority of the immuno-oncological research in HNC has been focused on ICIs, we mentioned also some studies exploring cell-based therapies and new discovery-based approaches. Immunotherapy for thyroid cancer and rare R/M HNCs, such as salivary glands, nasopharynx, paranasal sinus, and nuclear protein in testis (NUT) midline carcinoma is also reviewed.

\section{Standard of care}

The role of ICIs has been mainly investigated in the R/M setting of HNSCC [4, 5], both in platinum-resistant and platinum-sensitive diseases.

\section{Platinum-sensitive R/M HNSCC}

For nearly three decades, the mainstay of first-line palliative systemic chemotherapy (CT) has been cisplatin combined with 5-fluorouracil (5-FU) [6] or, more recently, with taxane [7] due to the increased response rate.

Food and Drug Administration (FDA) approved the anti-epidermal growth factor receptor (EGFR) monoclonal antibody cetuximab in 2006 for patients with HNSCC. When combined with platinum and 5-FU (the so-called "EXTREME" regimen), cetuximab significantly increased both progression-free survival [PFS, hazard ratio (HR) $=0.54,95 \%$ confidence interval (CI): 0.43-0.67] and overall survival (OS, $\mathrm{HR}=0.80,95 \%$ CI: 0.64-0.99) in patients with R/M disease representing until 2019 the standard-of-care for R/M HNSCC patients [8]. Alternative cetuximab plus platinum-based CT regimens include cisplatin + cetuximab +/paclitaxel (the B490 phase II Italian study) [9], and cisplatin + cetuximab + docetaxel (the so-called "TPEx" regimen explored in phase II French study) [10]. Whatever CT is associated with platinum, until 2019, the mainstay treatment for platinum-sensitive R/M HNSCC was a cetuximab + platinum-based CT. With this therapeutic approach, objective response rate (ORR) ranges from 36\% (EXTREME) to 59\% (TPEx), median PFS from 5.1-5.6 (EXTREME) to 6-7 months (B490), median OS from 10.1-10.7 (EXTREME) to 14.5 months (TPEx). In 2019, based on the results from KEYNOTE-048, both the FDA and the European Medicines Agency (EMA) approved the anti-PD-1 pembrolizumab (pembro) alone or with CT as first-line therapy in R/M HNSCC [11]. FDA-approved pembro with CT regardless of programmed death-ligand 1 (PD-L1) status, and pembro alone for patients with a combined positive score (CPS) PD-L1 $\geq 1$. By contrast, EMA approval was reserved for patients with CPS PD-L1 $\geq 1$ expressing tumors. So far, PD-L1 was the first predictive biomarker for treatment efficacy with PD-1 inhibitors in HNSCC.

The KEYNOTE-048 trial included 882 platinum-sensitive R/M HNSCC patients randomized 1:1:1 to receive pembro monotherapy, pembro plus cisplatin/carboplatin, and 5-FU versus the EXTREME regimen. Pembro significantly prolonged OS in patients with CPS $>1$ and $\geq 20$; irrespectively of PD-L1, pembro was non-inferior to the EXTREME regimen [12]. 
ORR and PFS were similar between pembro plus CT and the EXTREME regimen (ORR 36.3\% versus 35.6\%, PFS 5.1 versus 4.9 months, respectively). In comparison, a lower ORR was observed in those patients treated with pembro alone compared to patients treated with EXTREME $(23.3 \%$ versus $36.1 \%$ for CPS $\geq$ $20,19.1 \%$ versus $34.9 \%$ for CPS $\geq 1$ ). In patients treated with pembro monotherapy, prolonged duration of response (DoR) has been observed compared to standard of care (SoC) for patients with CPS $\geq 20$ and $\geq 1$ (23.4 versus 4.2 months, 24.8 versus 4.5 months, respectively). The benefit of pembro has been maintained for those patients with CPS 1-19, where the HR for OS demonstrated a slight advantage of pembro versus EXTREME and favored pembro + CT over EXTREME. In CPS-negative patients, HR for OS was 1.51 (95\% CI: 0.96-2.37) for pembro versus EXTREME and 1.21 (95\% CI: 0.76-1.94) for pembro + CT versus EXTREME, even if the analyses could have been limited by the small sample size of this group of patients [13]. Pembro alone demonstrated a more favorable safety profile with a lower incidence of treatment-related adverse events (TRAEs) equal to or higher than grade 3 TRAEs compared to EXTREME (17\% versus 69\%). No significant differences have been observed between pembro plus CT versus EXTREME (71\% versus $69 \%$ ). Based on these data, the society for immunotherapy of cancer published a consensus statement on immunotherapy for the treatment of HNSCC, where they suggested the use of a combination of ICI plus CT in patients requiring a quick tumor shrinkage such as those with more significant tumor burden and rapidly growing disease [14].

Despite the outstanding results of KEYNOTE-048, the response rate with ICIs is still modest. To intensify immune response, two studies (CheckMate-651 and Kestrel) are currently testing the efficacy of the combination of anti-cytotoxic T-lymphocyte-associated antigen 4 (CTLA-4) and anti-PD-L1 agents versus the EXTREME regimen for first-line R/M HNSCC.

The CheckMate-651 randomized (1:1) 947 patients to combined therapy with the anti-PD-1 nivolumab (nivo) and the anti-CTLA-4 ipilimumab versus the EXTREME regimen (cetuximab + 5-FU + cisplatin or carboplatin) [15] independently of PD-L1 status. The primary endpoint of this phase III study is OS in all comers and in PD-L1 positive patients. The final results of this study have been presented at European Society for Medical Oncology (ESMO) 2021 Annual Conference, and have shown that the immunotherapy combination did not provide a statistically significant improvement over EXTREME [15].

The KESTREL study was a randomized trial assessing three interventions: a combination of anti-CTLA-4 tremelimumab + anti-PD-L1 durvalumab versus durvalumab alone versus standard CT (EXTREME) [16]. In February 2021, a press release announced that the study did not meet its primary endpoint, which was OS improvement.

In conclusion, immunotherapy with the anti-PD-1 pembro (alone or in combination with $\mathrm{CT}$ ) is the current SoC for PD-L1 + platinum-sensitive R/M HNSCC.

\section{Platinum-resistant R/M HNSCC}

The prognosis is still dismal in loco-regionally advanced HNSCC patients recurring in the first months after platinum-containing curative treatments and in those progressing on platinum-based CT. In this setting, until 2016 the SoC was single agent CT with methotrexate or taxanes (docetaxel or paclitaxel), or with the single agent anti-EGFR cetuximab, especially in the USA. SoC provides a modest ORR (5.8\%), and prognosis is poor (median PFS 2.3 months, median OS 5.1\%) [5].

In platinum-resistant R/M HNSCC, several studies have explored the efficacy and the safety of ICIs. The two pivotal studies that led to the approval of anti-PD-1 agents pembro and nivo in this setting are the phase III KEYNOTE-040 [4] and CheckMate-141 [5] clinical trials. For nivo, the license from the regulatory agencies was not conditioned by PD-L1 expression. On the contrary, the EMA approved pembro only in those patients with R/M HNSCC expressing PD-L1 with a $\geq 50 \%$ tumor proportion score (TPS) and progressing on or after platinum-containing $\mathrm{CT}$.

The main results of CheckMate-141 and KEYNOTE 040 are reported in Table 1. 
Table 1. Trials of approved checkpoint inhibitors for R/M HNSCC

\begin{tabular}{|c|c|c|c|c|c|c|c|}
\hline Authors & Study & Setting & $\begin{array}{l}\text { Study } \\
\text { arm }\end{array}$ & $\begin{array}{l}\text { Control } \\
\text { arm }\end{array}$ & Median OS & & Approval \\
\hline \multirow{2}{*}{$\begin{array}{l}\text { Ferris et } \\
\text { al. [5] }\end{array}$} & \multirow[t]{2}{*}{ CheckMate-141 } & \multirow{2}{*}{$\begin{array}{l}\text { Platinum- } \\
\text { resistant }\end{array}$} & \multirow[t]{2}{*}{ Nivo } & \multirow[t]{2}{*}{ SoC } & \multicolumn{2}{|l|}{ Nivo: 7.5 months } & FDA \\
\hline & & & & & \multicolumn{2}{|l|}{ SoC: 5.1 months } & EMA \\
\hline \multirow{2}{*}{$\begin{array}{l}\text { Cohen } \\
\text { et al. [4] }\end{array}$} & \multirow[t]{2}{*}{ KEYNOTE-040 } & \multirow{2}{*}{$\begin{array}{l}\text { Platinum- } \\
\text { resistant }\end{array}$} & \multirow[t]{2}{*}{ Pembro } & \multirow[t]{2}{*}{ SoC } & \multicolumn{2}{|l|}{ Pembro: 8.4 months } & FDA \\
\hline & & & & & \multicolumn{2}{|l|}{ SoC: 6.9 months } & EMA $($ TPS $\geq 50 \%)$ \\
\hline \multirow{6}{*}{$\begin{array}{l}\text { Burtness } \\
\text { et al. [11] }\end{array}$} & \multirow[t]{6}{*}{ KEYNOTE-048* } & \multirow[t]{6}{*}{ First-line } & \multirow[t]{3}{*}{ Pembro } & \multirow[t]{3}{*}{ EXTREME } & $P D-L 1 C P S \geq 20$ & $P D-L 1 C P S \geq 1$ & FDA \\
\hline & & & & & Pembro: 14.9 months & Pembro: 12.3 months & EMA \\
\hline & & & & & EXTREME: 10.8 months & EXTREME: 10.4 months & \\
\hline & & & \multirow{3}{*}{$\begin{array}{l}\text { Pembro } \\
+\mathrm{CT}\end{array}$} & \multirow[t]{3}{*}{ EXTREME } & $P D-L 1 C P S \geq 20$ & $P D-L 1 C P S \geq 1$ & \\
\hline & & & & & $\begin{array}{l}\text { Pembro + CT: } 14.7 \\
\text { months }\end{array}$ & $\begin{array}{l}\text { Pembro + CT: } 13.6 \\
\text { months }\end{array}$ & \\
\hline & & & & & EXTREME: 11.1 months & EXTREME: 10.6 months & \\
\hline
\end{tabular}

${ }^{*}$ ESMO 2020 Congress update

The CheckMate-141 design was similar to that of the KEYNOTE-040, except for some main differences such as patient selection, dosages, schedules, and distribution of SoC regimens and treatments beyond progression [17]. The KEYNOTE-040 excluded patients recurring or progressing within three months of previous multimodal therapy containing platinum for locally advanced disease, and only $1.2 \%(N=6$ of 495$)$ of the HNSCC patients were heavily pre-treated ( $\geq 3$ lines of prior systemic therapy) as compared to $19.9 \%$ ( $N=72$ of 361) in the CheckMate-141. Among SoC chemotherapies, docetaxel was administered at $75 \mathrm{mg} / \mathrm{m}^{2}$ every 3 weeks (Q3W) in the KEYNOTE-040 trial, whereas in CheckMate-141 it was $30 \mathrm{mg} / \mathrm{m}^{2} \mathrm{QW}$. Moreover, the fraction of subjects pre-treated with docetaxel and cetuximab differed in the two studies. Those treated with prior docetaxel were $42 \%$ patients in the pembro study versus $21 \%$ in the nivo one, those with cetuximab were $30 \%$ versus $11 \%$, respectively. By contrast, in the CheckMate-141, there was a higher number of patients who received methotrexate as SoC (38\% versus $27 \%$ in KEYNOTE-040). Moreover, the percentage of patients in the $\mathrm{SoC}$ arm treated by ICIs beyond progression was different. Indeed, in the second-line pembro trial, an ICI was administered at disease progression in $12.5 \%$ of patients included in the SoC arm, potentially impacting on OS of these patients.

Other clinical trials studying different ICIs failed to demonstrate a significant improvement in the management of R/M HNSCC. The most relevant ones are the HAWK, CONDOR, EAGLE, and CheckMate-714.

The HAWK was a phase II single-arm trial assessing the activity of the anti-PD-L1 durvalumab in R/M HNSCC platinum-resistant patients with PD-L1 high expression (defined as patients with $\geq 25 \%$ of tumor cells expressing PD-L1). It included 112 patients, and the ORR of durvalumab was 16.2\% (95\% CI: 9.9-24.4), with higher responses in HPV-positive patients (29.4\% versus $10.9 \%$ HPV-negative). Minor safety issues were observed ( $8 \%$ grade 3 TRAEs). Survival was in line with that already described in other single-agent immunotherapy (median PFS and OS 2.1 and 7.1 months, respectively) [18].

The CONDOR trial included platinum-resistant PD-L1 negative R/M HNC patients. This was a phase II open-label study enrolling 267 patients that were randomized in 3 arms: 2 were similar to the KESTREL ones (durvalumab + tremelimumab; durvalumab), the third one explored anti-CTLA-4 alone (tremelimumab). The primary endpoint was ORR. The addition of tremelimumab failed to result in an increased ORR: 9.2\% with durvalumab, $1.6 \%$ with tremelimumab, and $7.8 \%$ with the combination. This modest response rate may be explained by the low expression of PD-L1, but the survival benefit was similar to what is usually reported in second-line treatments with anti-PD-1 [19]. The rate of grade 3-4 TRAEs was similar in three arms with $15.8 \%, 12.3 \%$, and $16.9 \%$, respectively.

The EAGLE was a randomized, open-label, phase III trial that included 736 platinum-resistant patients to receive second-line durvalumab alone, durvalumab + tremelimumab, or SoC (primary endpoint OS). Unfortunately, no increase in survival was observed. However, outcomes were similar to the ones found with anti-PD-1 agents nivo and pembro. Several reasons have been claimed to interpret the negative results. Patients with PD-L1 expressing tumor could be potentially under-represented in the durvalumab arm; 
tremelimumab is an IgG2 monoclonal antibody anti-CTLA-4 and, as such, unable to induce the antibodydependent cell cytotoxicity; moreover, we could not exclude a different activity of PD-1 and PD-L1 agents. Furthermore, it seems that the $\mathrm{SoC}$ arm has been somehow favored by the enrolment of more patients with Eastern Cooperative Oncology Group (ECOG) performance status (PS) 0 and with the checkpoint therapy administered in $36 \%$ of patients beyond progression [20]. Interestingly, peripheral blood tumor cell mutation burden (bTMB) proved to be a prognostic factor [21]. Based on that, bTMB has become the second predictive marker along with PD-L1 expression for the activity of checkpoint inhibitors.

The phase II randomized trial CheckMate-714 explored the anti-PD-1 nivo +/- the anti-CTLA-4 ipilimumab, with ORR as the primary endpoint [22]. Although final results are still pending, a press release from Bristol-Myers Squibb anticipated that this trial did not meet its primary endpoint.

The negative results of CONDOR, EAGLE, and CheckMate-714 trials hinted at the possible limitation of the activity of anti-CTLA-4 for platinum-resistant R/M HNSCC patients. At this stage, anti-PD-1 monotherapy with nivo or, where approved, pembro is indicated in this patient population as a single agent.

In conclusion, immunotherapy with the anti-PD- 1 nivo or pembro is the current SoC for platinumresistant R/M HNSCC, independently of PD-L1 expression.

\section{Discovery-based and a new generation of immune-oncology agents}

Different strategies have been hypothesized to improve the activity of ICI in HNCs, including approaches tackling tumor microenvironment (TME) and host factors. Indeed, despite a large number of trials, the global activity of immunotherapy is still weak and limited to a very selected patient population (PD-L1 positive and bTMB > 16 mutations/megabase) because of primary and secondary resistance. HNCs microenvironment is characterized by an immunosuppressive feature, and the early angiogenic switch is one of the significant contributors to the accumulation of immune suppressive cells in the TME [2]. Cancer growth is associated with hypoxia that, in turn, induces the production of vascular endothelial growth factor (VEGF) and cytokines [e.g., interleukin 1 (IL-1), IL-6, and IL-17]. VEGF binding to VEGF receptor 2 (VEGFR2) on Treg cells and myeloid-derived suppressor cells (MDSCs) participates in the maintenance of the immunosuppressive TME. Moreover, the hypoxic status contributes to the impairment of T-cell effectors and PD-L1 upregulation, providing a solid rationale in combining antiangiogenic agents with ICI. In this context, the association of lenvatinib (a multi-kinase inhibitor with a significant antiangiogenic activity) plus pembro is one of the most investigated in several malignant tumors. In a phase Ib/II trial, this association in unselected, heavily pretreated patients with multiple solid tumors resorted to an ORR of $36.4 \%$ in R/M HNSCC according to immune-related response evaluation criteria in solid tumors (RECIST) [23] with $91 \%$ of patients experienced a side effect higher than grade 3 [24]. These toxicities included adverse events (AEs) more specifically associated with antiangiogenic agents (e.g., arterial hypertension). However, some of the observed toxicities were known to be both immune-related and antiangiogenic-related (e.g., fatigue and diarrhea).

Based on this promising activity, a prospective phase III study is currently recruiting (LEAP-010) patients with R/M HNSCC, PD-L1 CPS $\geq 1$, to receive pembro plus lenvatinib $20 \mathrm{mg}$ or placebo first-line [25]. Several trials using the same approach are ongoing in R/M HNSCC (e.g., phase II trials with the anti-PD-1 pembro and ramucirumab; anti-PD-L1 atezolizumab plus bevacizumab) [26, 27].

The former study is made of two parts: the first is a de-escalation phase I trial of the combination of the anti-VEGFR2 monoclonal antibody ramucirumab + the anti-PD-1 pembro; in case of absence of safety concerns, and after defining the optimal drug dose, a second phase will be a single-arm phase II trial assessing the aforementioned combination [24]. The ATHENA study is a phase II trial exploring the activity and safety of the association of the anti-PD-L1 atezolizumab and the anti-VEGF-A/B monoclonal antibody bevacizumab in platinum-resistant R/M HNSCC [25]. In both studies, the primary endpoint is the ORR.

Other molecular actors of the TME are the focus of the current clinical research. One of the most relevant includes indoleamine deoxygenase (IDO), involved in tryptophan metabolism [28], contributing to the immunosuppressive features of the TME. ID01 is targeted by epacadostat. Within the KEYNOTE-037/ Echo-202 phase I/II trial, epacadostat plus pembro determined a stable disease (SD) as the best response 
in one HNSCC patient [29]. In this context, the association of epacadostat + pembro is under investigation in the KEYNOTE-669/Echo-304 study [30]. This is a phase III trial comparing the combination of epacadostat + pembro versus pembro alone versus the EXTREME regimen in the first-line setting. The estimated study completion date is July 2021. Although several studies have been put on hold due to the negative results from the phase III trial in melanoma, in principle, ID01 remains a very promising immunotherapeutic target. Tumor-associated macrophages (TAMs) [31] may be a crucial target for immunotherapy. They are in the hypoxic area of the tumor where they release VEGF and transforming growth factor- $\beta$ (TGF- $\beta$ ) that, in turn, attract more TAMs, amplifying this immunosuppressive loop. TAMs phenotype shapes their role within TME, with "M1-like macrophages" acting with pro-inflammatory activities and antitumor effect through IL-12, IL-23, and interferon (IFN) gamma. On the opposite, "M2-like macrophages" provide an immunosuppressive TME with inhibition of "M1-like macrophages", secretion of IL-10, TGF- $\beta$, VEGF, tumor necrosis factor alpha (TNF- $\alpha$ ), and induction of angiogenesis.

Inhibiting the colony-stimulating factor (CSF) 1 receptor (CSF1R) can inactivate TAMs, so leading to a more favorable anti-cancer TME. Indeed, CSF1R, the receptor of the soluble mediator CSF, is one of the main actors in determining M2 macrophage polarization [32]. However, the study exploring the CSF1R inhibitor PLX3397 was terminated early owed to the lack of evidence of clinical efficacy [33]. The immunosuppressive feature of TME is shared with other non-HNSCC solid tumors. Besides, the enrichment of specific cells population such as infiltrating NK, $\mathrm{CD}^{+} \mathrm{T}$ cells, and Tregs is characteristic of HNSCC TME. In fact, human leucocyte antigen (HLA)-E is overexpressed in HNSCCs, and it acts inhibiting NK and CD8 ${ }^{+}$ $\mathrm{T}$ cells via NK group 2 member A (NKG2A), paving the way to tailored immunotherapeutic approaches. Monalizumab is an ICI targeting NKG2A, which is expressed on subsets of NK. NKG2A blockade promotes immune response mediated by $\mathrm{NK}$ and $\mathrm{CD} 8^{+} \mathrm{T}$ cells, and when combined with cetuximab, it enhances $\mathrm{NK}$ cell antibody-dependent cell-mediated cetuximab-induced cytotoxicity by itself. The effective combination of monalizumab and cetuximab has been investigated in a multicentric phase Ib-II trial [34]. The results of the phase II cohort on 40 platinum-resistant patients showed an ORR of $27.5 \%$, with median PFS and OS of 4.5 and 8.5 months, respectively. A very promising ORR of $20 \%$ (95\% CI: 11-35) was reported in cohort II, including 40 platinum-resistant patients previously treated with PD-L1 inhibitors. PFS and OS were still immature at the time of data presentation [35]. Based on these results, the INTERLINK-1 phase III study [36], has recently started, comparing monalizumab plus cetuximab versus placebo plus cetuximab in patients with R/M HNSCCs who had received a prior ICI and platinum-based CT but not cetuximab. Survival is the primary aim.

In addition to the block of negative regulatory receptors, the enhancement of co-stimulatory signals using agonistic monoclonal antibodies and small molecules is under evaluation. For example, the benefit of feladilimab [GlaxoSmithKline 3359609 (GSK3359609)], an inducible T-cell co-stimulator (ICOS) agonist added to pembro was demonstrated in INDUCE-1 [37] with an ORR of 24\%, a disease control rate (DCR) of 68\%: median PFS was 4.2 months, median OS 13.1 months [38].

The INDUCE-3 was a randomized, double-blind, adaptive, phase II/III study of feladilimab or placebo in combination with pembro for first-line treatment of CPS $\geq 1 \mathrm{R} / \mathrm{M}$ HNSCC [39]. The primary study endpoints were OS and PFS. In April 2021, the trial sponsor communicated that a lack of benefit in terms of efficacy was observed in the first interim analysis. For this reason, GSK decided to stop the enrolment in the INDUCE-4 trial as well. This was a randomized, double-blind, adaptive, phase II/III study of feladilimab in combination with pembro and 5-FU-platinum CT versus placebo in combination with pembro plus 5-FU-platinum CT for first-line treatment of R/M HNSCC [40].

Further immune checkpoints have been recently selected as potential targets for therapeutic agents, mostly in combination with other already available drugs. Most of them are co-stimulatory molecules involved in immune response, so there is a solid rationale to explore the clinical activity of their inhibition. Among them there are lymphocyte-activation gene 3 (LAG3) [41], which is targeted by relatlimab [42, 43] and eftilagimod [44]; ITIM domain (TIGIT) [45], that is inhibited by tiragolumab, a drug under investigation in combination with anti-PD-L1 atezolizumab as first-line treatment for PD-L1 positive R/M HNSCC [46]; TNFR-related gene (GITR) [47], whose agonist is under investigation in a phase Ib trial of multivalent autophagosome vaccine with anti-PD-1 immunotherapy [48]. 
In the last years, the employ of bi-specific antibodies has been considered as well. They are usually made of one subunit that is active against two different molecular targets. The most relevant are mucin domain 3 (TIM3) [49], tackled by the PD-1/TIM3 bispecific antibody R07121661 [50] and TGF- $\beta$, a well known immunosuppressive mediator [51], that is co-inhibited by bintrafusp alfa, an anti-TGF- $\beta$ /anti-PD-1 agent that showed promising activity in HNSCC [52]; IL-2 [53] and fibroblast-activating protein (FAP) [54] are the targets of R06874281, a bispecific cytokine acting on tumor-associated fibroblasts, that induced one durable response in one HNSCC patient included in a phase I study [55].

Therapeutic vaccination is another strategy currently in use to enhance immune response. Whether HPV-related HNSCC viral antigens (e.g., E6/E7) represent a potential target for vaccination, for HPV-negative HNSCCs tumor associated-antigens (TAA) such as for example mucin 1 (MUC1), carcinoembryonic antigen (CEA), and melanoma-associated antigen-A3 (MAGEA3, unmutated antigens) should be considered [56-59]. Tumor-specific antigens (TSA), deriving from cancer driver mutations [e.g., tumor protein p53 (TP53), rat sarcoma (RAS)] or passenger mutations, are under exploitation for clinical benefit [60]. ISA101 is the most advance in clinical development in HPV-related HNSCCs. This is a synthetic long-peptide HPV-16 vaccine inducing HPV-specific T cells in patients with incurable HPV-16-related cancers [61]. In the ISA101-MDACC study combining ISA101 and the PD-1 inhibitor nivo, the ORR was $30 \%$ in subjects with HPV-16 positive OPC [62]. A promising association of anti-PD-1 and ISA101 is under current evaluation in a randomized, double-blind, placebo-controlled, phase II trial that is testing the combination of the ISA101b vaccine and cemiplimab in HPV-16-positive R/M OPC patients [63]. The same vaccine is under evaluation in combination with utomilumab (anti-CD137 immunostimulatory antibody) [64]. Several trials testing therapeutic vaccination in a different phase of development both in HPV-related and HPV-unrelated HNSCCs are recruiting [65].

To provide a general overview of the several ICIs cited in this article, Figure 1 summarizes the mechanisms of action of some of them. In conclusion, some of the most recent ICIs (e.g., anti-ICOS) failed to improve the outcomes of R/M HNSCC over SoC immunotherapy. However, there are many innovative approaches targeting the tumor immune microenvironment that are still under investigation, and some of them have shown promising activity.

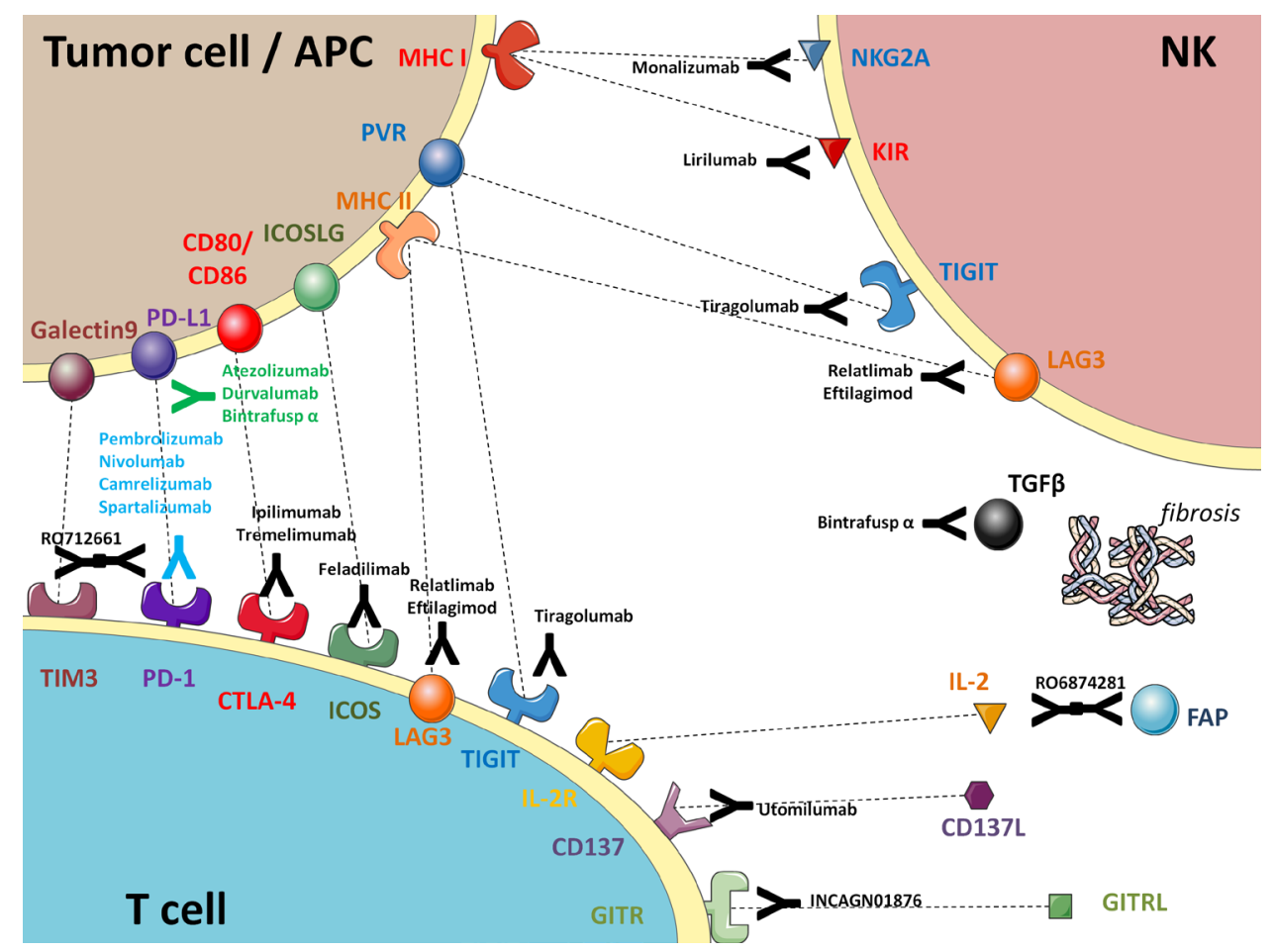

Figure 1. Schematic summary of approved and experimental immunotherapy agents in HNSCC (prepared using the open-access graphical repository of SMART-servier medical art). APC: antigen-presenting cell; MHC: major histocompatibility complex; PVR: poliovirus receptor; GITRL: the ligand for glucocorticoid-induced TNFR family-related protein; KIR: killer cell immunoglobulin-like receptors; IL2R: IL-2 receptors; ICOSLG: inducible T-cell costimulator ligand 


\section{Cell-based therapy: chimeric antigen receptor $\mathrm{T}$ cell and adoptive T-cell therapies}

Chimeric antigen receptor (CAR) $\mathrm{T}$ cell (CAR-T) therapy is based on $\mathrm{T}$ cells expressing an engineered receptor for a specific cancer antigen. CAR-T has been approved for B-cells malignancies such as mantle cells lymphoma, acute lymphoblastic leukemia, and large B cell lymphoma. This therapy is based on genetically modified autologous $\mathrm{T}$ cells expressing a CD19-specific CAR, lysing CD19-positive targets (normal and malignant B lineage cells).

The efficacy of CAR-T in solid tumors, particularly in HNCs, has to be still proved. Indeed, the main issues in this context are to find the suitable tumor antigen to tackle as CD19 for the hematological malignancies. For example, dysregulation of ErbB signaling has been involved in the pathogenesis of HNCs, potentially providing a candidate target for CAR-T therapy. A phase I/II trial with a gene-modified cellular therapy, called T4 immunotherapy, is currently ongoing in HNCs. These cells are engineered to co-express two transgenes: a CAR, T1E28z, targeted against a range of ErbB homodimers and heterodimers and a chimeric cytokine receptor, $4 \alpha \beta$, that allows the selective ex vivo expansion of engineered cells using IL-4. To limit the risk of side effects and in contrast to standard CAR-T therapy, engineered T-cells were delivered intratumor without prior lymphodepletion [66]. Preliminary data in 13 patients seem to confirm that intratumor administration of T4 immunotherapy is safe, being all TRAEs observed below or equal to grade 2, with no dose-limiting toxicities [common terminology criteria for adverse events (CTCAE) v4.0]. Despite the disease progression at study entry, an SD was observed in all 13 enrolled patients with the dosage $\geq 10 \times 10^{7} \mathrm{~T} 4^{+} \mathrm{T}$-cells [67]. A clinical trial involving patients with HPV-related head and neck and cervical cancer is currently recruiting [68]. The study tests engineered CAR-T that recognize the E6 HPV antigen. Twenty patients will be randomized to receive HPV-E6-specific CAR-T cells with or without anti-PD-1. Cross talk between PD-L1/PD-1 induces T cell exhaustion, and CAR-T cells armed with a PD-1 antagonist could potentially enhance the efficacy of CAR-T.

Different approaches with adoptive T-cell therapy have been investigated in EBV-related nasopharyngeal cancer (NPC). EBV-specific autologous polyclonal cytotoxic T lymphocytes (CTL) were administered with significant clinical and immunological responses in patients with relapsed/metastatic EBV-related NPC [69-72]. More recently, a clinical trial with CAR-T cells involving both NPC and breast cancer patients with epithelial cell adhesion molecule (EpCAM) CAR-T has been initiated. The study is currently still recruiting [73].

Only preclinical data on CAR-T have been published in salivary gland [74] and thyroid carcinomas [75].

As a general statement, the situation of CAR-T cells is much less advanced in HNSCC than in other malignancies (e.g., onco-hematologic ones). The recruitment of the ongoing studies still needs to be completed, and we anticipate that for at least some years HNC patients will not benefit from these innovative approaches, at least in clinical practice.

\section{Advanced thyroid and rare head and neck cancers}

The studies cited in previous paragraphs were conducted in R/M HNSCCs. In this setting, first-line pembro has become the first option for PD-L1 positive ( $C P S \geq 1$ ) disease, and second-line nivo is the best option for platinum-resistant R/M HNSCC. However, the pivotal trials that led to the regulatory approval of these ICIs did not include thyroid cancers and rare HNC. Since healthcare professionals devoted to HNC can face not only HNSCC, but also these tumor types, the following sections will concentrate on the role of immunotherapy in thyroid cancer and in salivary gland, nasopharyngeal, and paranasal sinuses carcinomas.

\section{Thyroid cancer}

Thyroid cancer (TC) can be classified in differentiated TC (DTC, including papillary TCs and follicular TCs: PTCs and FTCs, respectively), medullary TC (MTC), and anaplastic TC (ATC). DTC is usually treated with surgery and radiometabolic treatment. In radioactive-iodine (RAI) refractory DTC, the current SoC is the antiangiogenic multi-kinase inhibitor lenvatinib. With this drug, the ORR, median PFS, and median OS are $64.8 \%, 18.3$ months, and not reached respectively [76].

Recently, at American Society of Clinical Oncology (ASCO) 2021, the results of the COSMIC-311 phase III randomized trial showed the superiority of cabozantinib over placebo after lenvatinib failure in RAI-refractory DTC [77]: ORR was 15\% (versus $0 \%$ with placebo), and median PFS and OS were not reached. 
MTC is a neuroendocrine malignancy arising from the parafollicular C cells of the thyroid. In the non-operable or metastatic setting, standard treatments include the multi-kinase antiangiogenic inhibitors vandetanib and cabozantinib [78]. The latter provides a significant benefit, especially in MTCs harboring rearranged in transfection (RET) M918T mutation (ORR 34\%, median PFS 13.9 months, median OS 44.3 months) [79].

ATC is an aggressive disease, often not operable, with a dismal prognosis. Recently, the combination of B type rapidly accelerated fibrosarcoma oncogene (BRAF) and mitogen-activated protein kinase (MEK) inhibitors showed to be active and safe in BRAF V600E advanced ATC (ORR 69\%, 12-month PFS 79\%, 12-month OS 80\%) [80].

Unselected TC patient population seems to have a weak benefit from immunotherapy, except for more aggressive DTC histotypes and ATC. Indeed, the safety and the antitumor activity of pembro $10 \mathrm{mg} / \mathrm{kg}$ Q2W were demonstrated in patients with PD-L1-positive, advanced PTC, and FTC who were enrolled in the multi-cohort phase Ib KEYNOTE-028 trial. Confirmed partial response (PR) was observed in 2 patients with PTC out of 22 enrolled, for an ORR of 9\% (95\% CI: 1-29\%) with a median response duration of 20 and 8 months, respectively. Global clinical benefit rate (i.e., confirmed PR + SD $\geq 6$ months) was 50\% (95\% CI: $28-72 \%$ ) [81].

Among the different strategies enacted to improve the activity of PD-1/PD-L1 axis inhibitors, combination with other classes of agents (e.g., antiangiogenic compounds) or with anti-CTLA-4 is under evaluation. Lenvatinib $20 \mathrm{mg}$ plus pembro $200 \mathrm{mg}$ i.v. Q3W upfront in patients with radioiodine-refractory (RAIR) progressive DTCs has been recently reported at ASCO 2020. Eighteen (64\%) out of 28 evaluable patients had a PR, 32\% had SD. An overlapping PR rate (63\%) was reported with single-agent lenvatinib in the randomized trial [82], suggesting the lack of an addictive effect as upfront treatment, although patients were enrolled regardless of PD-L1 expression. The clinical benefit rate [complete response (CR) + PR + SD] was 96\%. The primary aim was not met (CRs rate), and 12-month PFS was 75\%, appearing favorable compared to single-agent lenvatinib (63\%) and pembro (36\%). The side effect profile was not negligible, with $83 \%$ of patients experiencing grade 3 and 4 toxicities [83]. This study intended to investigate salvage pembro in patients undergoing disease progression (PD) during lenvatinib. PR was 17\% with 11 months of PFS [7.1-not estimable (NE)], suggesting a role of pembro in overcoming lenvatinib resistance [84].

The combination of nivo $3 \mathrm{mg} / \mathrm{kg}$ Q2W plus ipilimumab $1 \mathrm{mg} / \mathrm{kg}$ Q6W was investigated, but it failed to meet the expectations in a series of unselected patients with RAIR-DTC $(N=37)$, MTC $(N=10)$, and ATC $(N=7)$ [85]. The ORR was $9.4 \%$ (95\% CI: $2-25 \%)$. A higher activity has been observed in Hurtle cell carcinoma and ATC patients. Indeed, anti-PD-1 agent spartalizumab alone demonstrated a RECIST ORR of 19\% (95\% CI: 8.6-34.1\%) in a series of unselected, heavily pretreated ATC patients. According to immune-related RECIST (irRECIST), the ORR was 24\% (95\% CI: 12.1-39.5\%), including three patients with a CR and seven with a PR. As expected, considering the poor prognosis of this tumor, the median PFS was 1.7 months. Median OS was 5.9 months ( $95 \%$ CI, 2.4 months-not reached), with $40 \%$ of patients alive at one year. Interestingly, a correlation with a better response and longer PFS and OS was reported in patients with a PD-L1 expression with median OS not yet reached in the subset of patients with PD-L1 > 1\% [86]. While the activity of spartalizumab alone in BRAF-mutated ATC patients was weak with an ORR of 8\% [87], the association of vemurafenib and cobimetinib with the anti-PD-L1 atezolizumab led to a significant OS increase at 12-months (median OS not yet reached, 95\% CI: 15.96-NE) with an ORR of 53\% including one patient with CR (6\%), supporting the employ of the triplets in BRAF mutated patients population [88]. Trials are currently ongoing to test the role of immunotherapy in advanced thyroid cancers [89]. Recently, at ASCO 2021 annual meeting Leboulleux and colleagues reported the results of the AcSé phase II study [90], a French multicenter clinical trial exploring the activity and safety of pembro in rare malignancies. In the RAI-refractory TC cohort, the ORR was $11.1 \%$ in DTC and $18.8 \%$ in ATC [90]. The authors concluded that such an ORR was "low in DTC and not negligible in ATC, but with a short DoR". Further studies are needed to assess the potential added value of immunotherapy to targeted therapy in advanced TC. 
In conclusion, for DTC and MTC targeted therapies are the current SoC, and it is unlikely that immunotherapy alone will replace them. On the contrary, for ATC the introduction of immunotherapy has led to a relevant benefit, complementing the one provided by BRAF and MEK inhibition.

\section{Salivary gland carcinomas}

Salivary gland carcinomas (SGCs) have a complex classification, including more than 20 distinct tumor types [91]. The treatment of R/M SGC mainly depends on the histology. As a general approach, it might be helpful to distinguish between adenoid cystic carcinoma (ACC) and non-ACC. The former usually undergo a watchful-waiting strategy or from loco-regional procedures when oligometastatic or paucisymptomatic (e.g., lung metastasectomies [92] or liver local therapies [93]). Symptomatic R/M ACC or cases with a high disease burden can be treated with the multi-kinase inhibitor lenvatinib (ORR 11.5-15.6\%, median PFS 9.1-17.5 months, median OS 27.5 months) [94, 95]. Non-ACCs include a very heterogeneous group of malignancies, and the description of the treatment of each of them is outside the topic of the current review. As a general concept, non-ACC are treated with cytotoxic CT (e.g., cisplatin and doxorubicin or carboplatin and taxanes), but chemo-free approaches can be envisaged when specifically druggable molecular alterations are found [96]. Some examples include combined androgen blockade for androgen-receptor overexpression (ORR 64.7\%, 3-year PFS 11.8\%, 5-year OS 19.3\%) [97], trastuzumab (ORR 70.2\%, median PFS 8.9 months, median OS 39.7 months) [98] or trastuzumab-emtansine (ORR 90\%), or trastuzumab-deruxtecan (ORR 47\%, median PFS 14.1 months) [99] with human epidermal growth factor receptor 2 (HER2) amplification (both can be found in salivary duct carcinoma (SDC), and in adenocarcinoma not otherwise specified), entrectinib or larotrectinib for tropomyosin receptor kinase (TRK) translocations (mammary-analog secretory carcinoma), etc.

In SGCs, a close correlation between histotype and immunologic microenvironment features has been recently demonstrated [100]. SDC has the most favorable immunogenomic profile, with a moderate tumor mutational burden (TMB) and moderate-to-high levels of microenvironmental inflammation. In contrast, ACCs have immunogenomic profiles that are less favorable for immunotherapy, with very low TMB and an immune-depleted microenvironment. Myoepithelial carcinomas had an intermediate immune profile. These differences might be exploited for a more personalized approach. ICIs are deemed to have limited activity in ACC, where PD-L1 is not expressed in contrast with the overexpression of PD-L2. R/M ACCs have a marked enrichment of several gene alterations, and distinct patterns of intratumoral genetic heterogeneity were observed [101], and few data about the activity of immune checkpoint blockade in SGCs are available. In this setting, the KEYNOTE-028 phase Ib trial included a small SGC cohort of 28 PD-L1 positive patients treated with P. The response was primarily observed in histotypes with a high PD-L1 expression, and higher mutational load, 3 PRs (11.5\%) have been reported in two adenocarcinomas nitric oxide synthase (NOS) and one case of high-grade serous carcinoma [102].

More recently, results from a multicentric retrospective study on 24 R/M SGCs treated with nivo showed ORR 4.2\%, but some patients achieved long-term disease control and a median PFS of 1.6 and median OS of 10.7 months. A biomarker analysis revealed significantly increased OS in patients with ECOG PS of 0, low neutrophil-to-lymphocyte ratio, lactate dehydrogenase, and C-reactive protein [103].

At ASCO 2021 annual meeting, Burman et al. [104] presented the final results of a phase II trial assessing the activity of nivo + ipilimumab in R/M SGCs. This study included non-ACC patients only and met its primary endpoint of improving the ORR. With combo-immunotherapy, ORR was $16 \%$, and the authors stated that the responses were durable. However, this benefit was observed only in high-grade non-ACC cases.

As in TC, also in SGC targeted therapies have been playing an increasing role. Further research needs to be conducted to confirm the role of immunotherapy in R/M SGCs.

\section{Nasopharyngeal carcinoma, paranasal cancers, NUT midline carcinoma}

NPC is distinct from other HNSCC due to the etiological differences (Epstein Barr virus-related in endemic areas) and its unique biological characteristics [105]. NPC is often diagnosed as a loco-regionally advanced disease, and in this setting, concomitant chemo-radiation is the mainstay treatment. Loco-regional treatments might be preceded or followed by induction or adjuvant CT, respectively [106]. In the R/M setting, the 
first-line therapy in the last five years has been cisplatin + gemcitabine. This combination CT provides an ORR of 64.1\%, and median PFS and OS are 7 and 29.1 months [107].

Preliminary results of a multi-institutional real-world experience of R/M NPC patients showed that ICIs have higher activity in EBV-related cancers [108]. EBV-related NPC cells expressed several viral proteins such as the EBV nuclear antigen 1 (EBNA1), latent membrane protein 1 (LMP1), LMP2A, and LMP2B [109]. These proteins induce a balanced viral replication to maintain the latency of viral infection. At the same time, this phenomenon reduces the possibility of the presentation of viral antigens to the immune system. Moreover, increased PD-L1 expression was associated with LMP1 [110].

Multiple clinical trials have demonstrated an ORR of about $20-25 \%$ in patients with platinum-refractory NPC with the single-agent activity of PD-1 inhibitors [111-113].

In a phase II study [114], nine out of 45 enrolled patients with R/M NPC received nivo with an ORR of 20.5\%, 1-year OS and PFS were 59\%, and 19.3\%, respectively. No relevant associations were found with PD-L1 expression, plasma EBV-DNA clearance, and survival. Patients with loss of either or both HLA-A or HLA-B expression had improved PFS [111].

Additionally, results from two single-arm, phase I trials examined camrelizumab, a PD-1 inhibitor, in the treatment of advanced solid tumors and R/M NPC alone and associated with CT. Safety and preliminary antitumor efficacy were reported for camrelizumab monotherapy in a dose-escalation phase I study, which enrolled subjects with advanced solid tumors who had failed current standard antitumor therapies [112]. This open-label, non-randomized, phase I study, evaluated the safety and efficacy of using the combination treatment of camrelizumab, gemcitabine, and cisplatin in R/M NPC [112]. In the camrelizumab monotherapy arm, with a median follow-up of 9.9 months, 34\% (95\% CI, 24-44\%) of evaluable patients experienced a confirmed objective response. Toxicities profile was acceptable, with $16 \%$ of patients experiencing TRAEs of grade 3 or 4 . For the combination arm, 91\% (95\% CI, 72-97\%) of evaluable patients achieved an objective response, with a severe concern about the safety profile with $87 \%$ of patients developing grade 3 TRAEs and $22 \%$ grade 4 TRAEs. This toxicity was mostly chemo-related since it was hematological. However, some immune-related AEs (e.g., skin rash) were observed as well, even though less frequently.

The CAPTAIN study, a phase II trial presented at ESMO Virtual Congress 2020, assessed the safety and activity of camrelizumab in R/M NPC who had progressed after more than two lines of CT, with an ORR of 28.2\% (95\% CI: 21.3-36.0), median PFS and OS of 3.7 and 17.1 months, respectively. The safety profile was manageable, with toxicities consistent with previous reports with camrelizumab [113].

Recently, Yang et al. [115] reported the results of the CAPTAIN-1st study, a multicenter, randomized, double-blind, phase III trial assessing the combination of cisplatin + gemcitabine + camrelizumab versus cisplatin + gemcitabine + placebo in R/M NPC. This study showed a relevant improvement of PFS with camrelizumab (median PFS 10.8 months versus 6.9 months with placebo, $P<0.0001$ ), with a significant ORR (88.1\%). The impressive results of the CAPTAIN-1st trial could pave the way for a new first-line treatment for R/M NPC, which shall be chemo-immunotherapy.

Sinonasal epithelial cancers (SNCs) are treated with multimodal treatments, including induction CT and loco-regional approaches [116]. R/M SNC has a poor prognosis (median OS 13 months) [117], and it is usually treated with cytotoxic CT.

Immunotherapy could have a potential role in SNCs, due to its strong association, specifically in undifferentiated sinonasal cancers (SNUC), with immune system-related functional pathways which have been found to contain a high proportion of $\mathrm{CD}^{+}$effector memory cells in their microenvironment. The identification of immune pathways should be further investigated, for possible integration of immunotherapy into the multidisciplinary approach to these cancers [118], because no data are available at the moment on the activity of checkpoint inhibitors in clinical practice.

NUT carcinoma (or NUT midline carcinoma) is a rare and aggressive subtype of epithelial carcinoma, defined by the evidence of NUT gene (NUTM1) rearrangement. Common sites include the head and neck region (in particular the nasal cavity and paranasal sinuses) and mediastinum $[119,120]$. 
No clinical evidence in the literature has been reported yet about a possible role of ICIs in this setting; however, the efficacy is under evaluation within clinical trials in other sites of the same histology. For instance, the CHANCE trial is aiming to explore the antitumor activity and the safety profile of atezolizumab in pretreated advanced non-small cell lung cancer (NSCLC) patients with rare histological subtypes, including lung NUT-carcinomas [121].

\section{Conclusion}

The landscape of immunotherapy in HNSCC, NPC, SGC, and TC is constantly evolving. This review summarizes the main trial results in these malignant tumors.

In the context of R/M HNSCC treatment, checkpoint inhibitors have changed the state of the art, finally improving patients outcome, even if only a minority of HNSCC patients may benefit from immunotherapy. In the loco-regionally advanced setting, the role of ICIs, especially in combination with radiotherapy or as neoadjuvant/adjuvant agents for surgery is still under study.

To date, the only predictive factors are PD-L1 expression and TMB, and other biological markers are under investigation. The field of research is currently focused on, bringing the benefits of ICI to a more significant portion of patients including rare HNC histotypes. Indeed, promising results have already been reported in SGC, NPC, and TC.

\section{Abbreviations}

5-FU: 5-fluorouracil

ACC: adenoid cystic carcinoma

ASCO: American Society of Clinical Oncology

ATC: anaplastic thyroid cancer

BRAF: B type rapidly accelerated fibrosarcoma oncogene

bTMB: blood tumor cell mutation burden

CAR: chimeric antigen receptor

CAR-T: chimeric antigen receptor $\mathrm{T}$ cell

CI: confidence interval

CPS: combined positive score

CR: complete response

CSF: colony-stimulating factor

CSF1R: colony-stimulating factor 1 receptor

CT: chemotherapy

CTLA-4: cytotoxic T-lymphocyte-associated antigen 4

DTC: differentiated thyroid cancer

EBV: Epstein-Barr virus

EMA: European Medicines Agency

ESMO: European Society for Medical Oncology

FDA: Food and Drug Administration

HLA: human leucocyte antigen

HNC: head and neck cancers

HNSCC: head and neck squamous cell carcinoma

HPV: human papillomavirus

HR: hazard ratio 
ICIs: immune checkpoint inhibitors

ICOS: inducible T-cell co-stimulator

IDO: indoleamine deoxygenase

IL-1: interleukin 1

LMP1: latent membrane protein 1

MHC: major histocompatibility complex

MTC: medullary thyroid cancer

nivo: nivolumab

NK: natural killer

NKG2A: natural killer group 2 member A

NPC: nasopharyngeal cancer

NUT: nuclear protein in testis

OPC: oropharyngeal cancer

ORR: objective response rate

OS: overall survival

PD-1: mprogrammed cell death protein 1

PD-L1: programmed death-ligand 1

pembro: pembrolizumab

PR: partial response

PS: performance status

PTCs: papillary thyroid cancers

Q3W: every 3 weeks

$\mathrm{R} / \mathrm{M}$ : recurrent/metastatic

RAI: radioactive-iodine

RECIST: related response evaluation criteria in solid tumors

SD: stable disease

SDC: salivary duct carcinoma

SGCs: salivary gland carcinomas

SoC: standard of care

TAMs: tumor-associated macrophages

TC: thyroid cancer

TGF- $\beta$ : transforming growth factor- $\beta$

TMB: tumor mutational burden

TME: tumor microenvironment

TPEx: cisplatin + cetuximab + docetaxel

TRAEs: treatment-related adverse events

VEGF: vascular endothelial growth factor

\section{Declarations}

Author contributions

SC, DMF and LDL contributed conception and design of the study; DMF, AO and SC organized the tables; SC, DMF and LL wrote the first draft of the manuscript. All authors contributed to manuscript drafting, writing, and revision and fulfilled the criteria included in the ICMJE guidelines. All authors read and approved the submitted version. 


\section{Conflicts of interest}

Laura D. Locati reports grant and other financial relationships with Eisai, Ipsen, Merck Serono, MSD, BMS, Lilly. Lisa Licitra declares the following conflicts of interests: receipt of grants/research supports (Funds received by my institution for clinical studies and research activities in which I am involved) from AstraZeneca, BMS, Boehringer Ingelheim, Celgene International, Debiopharm International SA, Eisai, Exelixis Inc, Hoffmann-La Roche Ltd, IRX Therapeutics inc, Medpace Inc, Merck-Serono, MSD, Novartis, Pfizer, Roche; receipt of honoraria or consultation fees (for public speaking/teaching in medical meetings and/or for expert opinion in advisory boards) from AstraZeneca, Bayer, BMS, Eisai, MSD, Merck-Serono, Boehringer Ingelheim, Novartis, Roche, Debiopharm International SA, Sobi, Ipsen, Incyte Biosciences Italy SRL, Doxa Pharma, Amgen, Nanobiotics Sa, and GSK. The remaining authors declare no conflicts of interest.

\section{Ethical approval}

Not applicable.

\section{Consent to participate}

Not applicable.

\section{Consent to publication}

Not applicable.

\section{Availability of data and materials}

Not applicable.

\section{Funding}

Not applicable.

\section{Copyright}

(c) The Author(s) 2021.

\section{References}

1. Mandal R, Şenbabaoğlu Y, Desrichard A, Havel JJ, Dalin MG, Riaz N, et al. The head and neck cancer immune landscape and its immunotherapeutic implications. JCI Insight. 2016;1:e89829.

2. Merlano MC, Denaro N, Garrone O. Immune escape mechanisms in head and neck squamous cell carcinoma and implication for new immunotherapy approach. Curr Opin Oncol. 2020;32:203-9.

3. Badoual C, Hans S, Merillon N, Van Ryswick C, Ravel P, Benhamouda N, et al. PD-1-expressing tumor-infiltrating T cells are a favorable prognostic biomarker in HPV-associated head and neck cancer. Cancer Res. 2013;73:128-38.

4. Cohen EEW, Soulières D, Le Tourneau C, Dinis J, Licitra L, Ahn MJ, et al; KEYNOTE-040 investigators. Pembrolizumab versus methotrexate, docetaxel, or cetuximab for recurrent or metastatic head-andneck squamous cell carcinoma (KEYNOTE-040): a randomised, open-label, phase 3 study. Lancet. 2019;393:156-67.

5. Ferris RL, Blumenschein G Jr, Fayette J, Guigay J, Colevas AD, Licitra L, et al. Nivolumab for recurrent squamous-cell carcinoma of the head and neck. N Engl J Med. 2016;375:1856-67.

6. Forastiere AA, Metch B, Schuller DE, Ensley JF, Hutchins LF, Triozzi P, et al. Randomized comparison of cisplatin plus fluorouracil and carboplatin plus fluorouracil versus methotrexate in advanced squamous-cell carcinoma of the head and neck: a southwest oncology group study. J Clin Oncol. 1992;10:1245-51.

7. Gibson MK, Li Y, Murphy B, Hussain MH, DeConti RC, Ensley J, et al; Eastern Cooperative Oncology Group. Randomized phase III evaluation of cisplatin plus fluorouracil versus cisplatin plus paclitaxel in 
advanced head and neck cancer (E1395): an intergroup trial of the eastern cooperative oncology group. J Clin Oncol. 2005;23:3562-7.

8. Vermorken JB, Mesia R, Rivera F, Remenar E, Kawecki A, Rottey S, et al. Platinum-based chemotherapy plus cetuximab in head and neck cancer. N Engl J Med. 2008;359:1116-27.

9. Bossi P, Miceli R, Locati LD, Ferrari D, Vecchio S, Moretti G, et al. A randomized, phase 2 study of cetuximab plus cisplatin with or without paclitaxel for the first-line treatment of patients with recurrent and/or metastatic squamous cell carcinoma of the head and neck. Ann Oncol. 2017;28:2820-6.

10. Guigay J, Aupérin A, Fayette J, Saada-Bouzid E, Lafond C, Taberna M, et al; GORTEC; AIO; TTCC, and UniCancer Head and Neck groups. Cetuximab, docetaxel, and cisplatin versus platinum, fluorouracil, and cetuximab as first-line treatment in patients with recurrent or metastatic head and neck squamous-cell carcinoma (GORTEC 2014-01 TPExtreme): a multicentre, open-label, randomised, phase 2 trial. Lancet Oncol. 2021;22:463-75.

11. Burtness B, Harrington KJ, Greil R, Soulières D, Tahara M, de Castro G Jr, et al; KEYNOTE-048 Investigators. Pembrolizumab alone or with chemotherapy versus cetuximab with chemotherapy for recurrent or metastatic squamous cell carcinoma of the head and neck (KEYNOTE-048): a randomised, open-label, phase 3 study. Lancet. 2019;394:1915-28.

12. Greil R, Rischin D, Harrington KJ, Soulières D, Tahara M, de Castro G, et al. 915MO long-term outcomes from KEYNOTE-048: pembrolizumab (pembro) alone or with chemotherapy (pembro $+\mathrm{C}$ ) vs EXTREME (E) as first-line $(1 \mathrm{~L})$ therapy for recurrent/metastatic $(\mathrm{R} / \mathrm{M})$ head and neck squamous cell carcinoma (HNSCC). Ann Oncol. 2020;31:S660-1.

13. Burtness B, Rischin D, Greil R, Soulières D, Tahara M, de Castroet G, et al. Abstract LB-258: efficacy of first-line (1 L) pembrolizumab by PD-L1 combined positive score $<1,1-19$, and $\geq 20$ in recurrent and/or metastatic (R/M) head and neck squamous cell carcinoma (HNSCC): KEYNOTE-048 subgroup analysis. Cancer Res. 2020;80:LB-258-LB-258.

14. Argiris A, Gillison M, Ferris RL, Harrington K, Sanchez TK, Baudelet C, et al. A randomized, open-label, phase 3 study of nivolumab in combination with ipilimumab vs extreme regimen (cetuximab + cisplatin/ carboplatin + fluorouracil) as first-line therapy in patients with recurrent or metastatic squamous cell carcinoma of the head and neck-CheckMate 651. Ann Oncol. 2016;27:vi350.

15. Argiris A, Harrington K, Tahara M, Ferris RL, Gillison M, Fayette J, et al. LBA36-Nivolumab (N) + ipilimumab (I) vs EXTREME as first-line (1 L) treatment (tx) for recurrent/metastatic squamous cell carcinoma of the head and neck (R/M SCCHN): final results of CheckMate 651. Ann Oncol. 2021;32: S1283-346.

16. Seiwert TY, Weiss J, Baxi SS, Ahn MJ, Fayette J, Gillison ML, et al. A phase 3, randomized, open-label study of first-line durvalumab (MEDI4736) \pm tremelimumab versus standard of care (SoC; EXTREME regimen) in recurrent/metastatic (R/M) SCCHN: KESTREL. J Clin Oncol. 2016;34:TPS6101.

17. Pai SI, Faivre S, Licitra L, Machiels JP, Vermorken JB, Bruzzi P, et al. Comparative analysis of the phase III clinical trials of anti-PD1 monotherapy in head and neck squamous cell carcinoma patients (CheckMate 141 and KEYNOTE 040). J Immunother Cancer. 2019;7:96.

18. Zandberg DP, Algazi AP, Jimeno A, Good JS, Fayette J, Bouganim N, et al. Durvalumab for recurrent or metastatic head and neck squamous cell carcinoma: results from a single-arm, phase II study in patients with $\geq 25 \%$ tumour cell PD-L1 expression who have progressed on platinum-based chemotherapy. Eur J Cancer. 2019;107:142-52.

19. Siu LL, Even C, Mesía R, Remenar E, Daste A, Delord JP, et al. Safety and efficacy of durvalumab with or without tremelimumab in patients with PD-L1-low/negative recurrent or metastatic HNSCC: the phase 2 CONDOR randomized clinical trial. JAMA Oncol. 2019;5:195-203.

20. Merlano MC. The EAGLE study: two is not always better than one. Ann Oncol. 2020;31:830-1. 
21. Ferris RL, Haddad R, Even C, Tahara M, Dvorkin M, Ciuleanu TE, et al. Durvalumab with or without tremelimumab in patients with recurrent or metastatic head and neck squamous cell carcinoma: EAGLE, a randomized, open-label phase III study. Ann Oncol. 2020;31:942-50.

22. Study of nivolumab in combination with ipilimumab versus nivolumab in combination with ipilimumab placebo in patients with recurrent or metastatic squamous cell carcinoma of the head and neck (CheckMate 714) [Internet]. USA: National Library of Medicine; c2021 [cited 2020 Dec 14]. Available from: https://clinicaltrials.gov/ct2/show/NCT02823574

23. Seymour L, Bogaerts J, Perrone A, Ford R, Schwartz LH, Mandrekar S, et al; RECIST working group. iRECIST: guidelines for response criteria for use in trials testing immunotherapeutics. Lancet Oncol. 2017;18:e143-52.

24. Taylor MH, Rasco DW, Brose MS, Vogelzang NJ, Richey SL, Cohn AL, et al. A phase $1 \mathrm{~b} / 2$ trial of lenvatinib plus pembrolizumab in patients with squamous cell carcinoma of the head and neck. J Clin Oncol. 2018;36:6016.

25. A study of pembrolizumab (MK-3475) with or without lenvatinib (E7080/MK-7902) as first line $(1 \mathrm{~L})$ intervention in a programmed cell death-ligand 1 (PD-L1) selected population with recurrent or metastatic head and neck squamous cell carcinoma (R/M HNSCC) (LEAP-010) (MK-7902-010) [Internet]. USA: National Library of Medicine; c2021 [cited 2020 Dec 14]. Available from: https:// clinicaltrials.gov/ct2/show/NCT04199104

26. Ramucirumab + pembrolizumab in patients with recurrent/metastatic head and neck squamous cell carcinoma [Internet]. USA: National Library of Medicine; c2021 [cited 2021 Mar 4]. Available from: https://clinicaltrials.gov/ct2/show/NCT03650764

27. Atezolizumab and bevacizumab in patients with recurrent or metastatic squamous-cell carcinoma of the head and neck (ATHENA) [Internet]. USA: National Library of Medicine; c2021 [cited 2021 Mar 4]. Available from: https://clinicaltrials.gov/ct2/show/NCT03818061

28. Yentz S, Smith D. Indoleamine 2,3-dioxygenase (IDO) inhibition as a strategy to augment cancer immunotherapy. BioDrugs. 2018;32:311-7.

29. Mitchell TC, Hamid O, Smith DC, Bauer TM, Wasser JS, Olszanski AJ, et al. Epacadostat plus pembrolizumab in patients with advanced solid tumors: phase I results from a multicenter, open-label phase I/II trial (ECHO-202/KEYNOTE-037). J Clin Oncol. 2018;36:3223-30.

30. Pembrolizumab plus epacadostat, pembrolizumab monotherapy, and the EXTREME regimen in recurrent or metastatic head and neck squamous cell carcinoma (KEYNOTE-669/ECHO-304) [Internet]. USA: National Library of Medicine; c2021 [cited 2021 Apr 1]. Available from: https://clinicaltrials.gov/ ct2/show/NCT03358472

31. Cannarile MA, Weisser M, Jacob W, Jegg AM, Ries CH, Rüttinger D. Colony-stimulating factor 1 receptor (CSF1R) inhibitors in cancer therapy. J Immunother Cancer. 2017;5:53.

32. Evrard D, Szturz P, Tijeras-Raballand A, Astorgues-Xerri L, Abitbol C, Paradis V, et al. Macrophages in the microenvironment of head and neck cancer: potential targets for cancer therapy. Oral Oncol. 2019;88:29-38.

33. A combination clinical study of PLX3397 and pembrolizumab to treat advanced melanoma and other solid tumors [Internet]. USA: National Library of Medicine; c2021 [cited 2021 Apr 1]. Available from: https://clinicaltrials.gov/ct2/show/NCT02452424

34. Study of monalizumab and cetuximab in patients with recurrent or metastatic squamous cell carcinoma of the head and neck [Internet]. USA: National Library of Medicine; c2021 [cited 2021 Apr 1]. Available from: https://clinicaltrials.gov/ct2/show/NCT02643550

35. Cohen RB, Bauman JR, Salas S, Colevas AD, Even C, Cupissol D, et al. Combination of monalizumab and cetuximab in recurrent or metastatic head and neck cancer patients previously treated with platinum-based chemotherapy and PD-(L)1 inhibitors. J Clin Oncol. 2020;38:6516. 
36. Assessment of efficacy and safety of monalizumab plus cetuximab compared to placebo plus cetuximab in recurrent or metastatic head and neck cancer (INTERLINK-1) [Internet]. USA: National Library of Medicine; c2021 [cited 2021 Apr 1]. Available from: https://clinicaltrials.gov/ct2/show/NCT04590963

37. Dose escalation and expansion study of GSK3359609 in participants with selected advanced solid tumors (INDUCE-1) [Internet]. USA: National Library of Medicine; c2021 [cited 2021 Apr 1]. Available from: https://clinicaltrials.gov/ct2/show/NCT02723955

38. Le Tourneau C, Rischin D, Groenland S, Lim A, Martin-Liberal J, Moreno V, et al. 10 inducible T cell co-stimulatory (ICOS) receptor agonist, GSK3359609 (GSK609) alone and combination with pembrolizumab: preliminary results from INDUCE-1 expansion cohorts in head and neck squamous cell carcinoma (HNSCC). Ann Oncol. 2020;31:S1.

39. Study of GSK3359609 and pembrolizumab in programmed death receptor 1-ligand 1 (PD-L1) positive recurrent or metastatic head and neck squamous cell carcinoma (INDUCE-3) [Internet]. USA: National Library of Medicine; c2021 [cited 2020 Dec 14]. Available from: https://clinicaltrials.gov/ct2/show/ NCT04128696

40. Study of GSK3359609 with pembrolizumab and 5-fluorouracil (5-FU)-platinum chemotherapy in participants with recurrent or metastatic head and neck squamous cell carcinoma (INDUCE-4) [Internet]. USA: National Library of Medicine; c2021 [cited 2020 Dec 14]. Available from: https:// clinicaltrials.gov/ct2/show/NCT04428333

41. Andrews LP, Marciscano AE, Drake CG, Vignali DA. LAG3 (CD223) as a cancer immunotherapy target. Immunol Rev. 2017;276:80-96.

42. Study of safety and tolerability of nivolumab treatment alone or in combination with relatlimab or ipilimumab in head and neck cancer [Internet]. USA: National Library of Medicine; c2021 [cited 2021 Apr 1]. Available from: https://clinicaltrials.gov/ct2/show/NCT04080804

43. Personalized immunotherapy in patients with recurrent/metastatic SCCHN that have progressed on prior immunotherapy [Internet]. USA: National Library of Medicine; c2021 [cited 2021 Apr 1]. Available from: https://clinicaltrials.gov/ct2/show/NCT04326257

44. Combination study with soluble LAG-3 fusion protein eftilagimod alpha (IMP321) and pembrolizumab in patients with previously untreated unresectable or metastatic NSCLC, or recurrent PD-X refractory NSCLC or with recurrent or metastatic HNSCC [Internet]. USA: National Library of Medicine; c2021 [cited 2021 Apr 1]. Available from: https://clinicaltrials.gov/ct2/show/NCT03625323

45. Harjunpää H, Guillerey C. TIGIT as an emerging immune checkpoint. Clin Exp Immunol. 2020;200:108-19.

46. A study of atezolizumab plus tiragolumab and atezolizumab plus placebo as first-line treatment in participants with recurrent/metastatic PD-L1 positive squamous cell carcinoma of the head and neck [Internet]. USA: National Library of Medicine; c2021 [cited 2021 Apr 1]. Available from: https:// clinicaltrials.gov/ct2/show/NCT04665843

47. Zappasodi R, Sirard C, Li Y, Budhu S, Abu-Akeel M, Liu C, et al. Rational design of anti-GITR-based combination immunotherapy. Nat Med. 2019;25:759-66.

48. Phase Ib trial of multivalent autophagosome vaccine with or without GITR agonist, with anti-PD-1 immunotherapy in HNSCC [Internet]. USA: National Library of Medicine; c2021 [cited 2021 Apr 1]. Available from: https://clinicaltrials.gov/ct2/show/NCT04470024

49. Wolf Y, Anderson AC, Kuchroo VK. TIM3 comes of age as an inhibitory receptor. Nat Rev Immunol. 2020;20:173-85.

50. A dose escalation and expansion study of R07121661, a PD-1/TIM-3 bispecific antibody, in participants with advanced and/or metastatic solid tumors [Internet]. USA: National Library of Medicine; c2021 [cited 2021 Apr 1]. Available from: https://clinicaltrials.gov/ct2/show/NCT03708328

51. Oshimori N, Oristian D, Fuchs E. TGF- $\beta$ promotes heterogeneity and drug resistance in squamous cell carcinoma. Cell. 2015;160:963-76. 
52. Cho BC, Daste A, Ravaud A, Salas S, Isambert N, McClay E, et al. Bintrafusp alfa, a bifunctional fusion protein targeting TGF- $\beta$ and PD-L1, in advanced squamous cell carcinoma of the head and neck: results from a phase I cohort. J Immunother Cancer. 2020;8:e000664.

53. Wrangle JM, Patterson A, Johnson CB, Neitzke DJ, Mehrotra S, Denlinger CE, et al. IL-2 and beyond in cancer immunotherapy. J Interferon Cytokine Res. 2018;38:45-68.

54. Kuzet SE, Gaggioli C. Fibroblast activation in cancer: when seed fertilizes soil. Cell Tissue Res. 2016;365:607-19.

55. Soerensen MM, Ros W, Rodriguez-Ruiz ME, Robbrecht D, Rohrberg KS, Martin-Liberal J, et al. Safety, PK/PD, and anti-tumor activity of R06874281, an engineered variant of interleukin-2 (IL-2v) targeted to tumor-associated fibroblasts via binding to fibroblast activation protein (FAP). J Clin Oncol. 2018;36:e15155.

56. Weed DT, Zilio S, Reis IM, Sargi Z, Abouyared M, Gomez-Fernandez CR, et al. The reversal of immune exclusion mediated by tadalafil and an anti-tumor vaccine also induces PDL1 upregulation in recurrent head and neck squamous cell carcinoma: interim analysis of a phase I clinical trial. Front Immunol. 2019;10:1206.

57. An open label phase I study to eval the safety and tolerability of a vaccine (GI-6207) consisting of whole, heat-killed recombinant saccharomyces cerevisiae (yeast) genetically modified to express CEA protein in adults with metastatic CEA-expressing ... [Internet]. USA: National Library of Medicine; c2021 [cited 2021 Apr 1]. Available from: https://clinicaltrials.gov/ct2/show/NCT00924092

58. Vaccine therapy in treating patients with advanced or metastatic cancer [Internet]. USA: National Library of Medicine; c2021 [cited 2021 Apr 1]. Available from: https://clinicaltrials.gov/ct2/show/ NCT00027534

59. 0804 GCC: MAGE-A3/HPV 16 vaccine for squamous cell carcinoma of the head and neck [Internet]. USA: National Library of Medicine; c2021 [cited 2021 Apr 1]. Available from: https://clinicaltrials.gov/ct2/ show/NCT00257738

60. von Witzleben A, Wang C, Laban S, Savelyeva N, Ottensmeier CH. HNSCC: tumour antigens and their targeting by immunotherapy. Cells. 2020;9:2103.

61. Kenter GG, Welters MJ, Valentijn AR, Lowik MJ, Berends-van der Meer DM, Vloon AP, et al. Vaccination against HPV-16 oncoproteins for vulvar intraepithelial neoplasia. N Engl J Med. 2009;361:1838-47.

62. Massarelli E, William W, Johnson F, Kies M, Ferrarotto R, Guo M, et al. Combining immune checkpoint blockade and tumor-specific vaccine for patients with incurable human papillomavirus 16-related cancer: a phase 2 clinical trial. JAMA Oncol. 2019;5:67-73.

63. A phase II study of cemiplimab and ISA101b in patients with recurrent/metastatic HPV16 positive OPC [Internet]. USA: National Library of Medicine; c2021 [cited 2020 Dec 14]. Available from: https:// clinicaltrials.gov/ct2/show/NCT04398524

64. Utomilumab and ISA101b vaccination in patients with HPV-16-positive incurable oropharyngeal cancer [Internet]. USA: National Library of Medicine; c2021 [cited 2021 Apr 1]. Available from: https:// clinicaltrials.gov/ct2/show/NCT03258008

65. Wang C, Dickie J, Sutavani RV, Pointer C, Thomas GJ, Savelyeva N. Targeting head and neck cancer by vaccination. Front Immunol. 2018;9:830.

66. Papa S, van Schalkwyk M, Maher J. Clinical evaluation of ErbB-targeted CAR T-cells, following intracavity delivery in patients with ErbB-expressing solid tumors. Methods Mol Biol. 2015;1317:365-82.

67. Papa S, Adami A, Metoudi M, Achkova D, van Schalkwyk M, Pereira AP, et al. A phase I trial of T4 CAR T-cell immunotherapy in head and neck squamous cancer (HNSCC). J Clin Oncol. 2018;36:3046.

68. HPV-E6-specific anti-PD1 TCR-T cells in the treatment of HPV-positive NHSCC or cervical cancer [Internet]. USA: National Library of Medicine; c2021 [cited 2021 Mar 4]. Available from: https:// clinicaltrials.gov/ct2/show/NCT03578406 
69. Comoli P, Pedrazzoli P, Maccario R, Basso S, Carminati O, Labirio M, et al. Cell therapy of stage IV nasopharyngeal carcinoma with autologous Epstein-Barr virus-targeted cytotoxic T lymphocytes. J Clin Oncol. 2005;23:8942-9.

70. Straathof KC, Bollard CM, Popat U, Huls MH, Lopez T, Morriss MC, et al. Treatment of nasopharyngeal carcinoma with Epstein-Barr virus-specific T lymphocytes. Blood. 2005;105:1898-904.

71. Secondino S, Zecca M, Licitra L, Gurrado A, Schiavetto I, Bossi P, et al. T-cell therapy for EBV-associated nasopharyngeal carcinoma: preparative lymphodepleting chemotherapy does not improve clinical results. Ann Oncol. 2012;23:435-41.

72. Renaud S, Lefebvre A, Mordon S, Moralès 0 , Delhem N. Novel therapies boosting T cell immunity in epstein Barr Virus-associated nasopharyngeal carcinoma. Int J Mol Sci. 2020;21:4292.

73. EpCAM CAR-T for treatment of nasopharyngeal carcinoma and breast cancer [Internet]. USA: National Library of Medicine; c2021 [cited 2021 Mar 4]. Available from: https://clinicaltrials.gov/ct2/show/ NCT02915445

74. Makita Y, Kunii N, Sakurai D, Ihara F, Motohashi S, Suzuki A, et al. Activated iNKT cells enhance the anti-tumor effect of antigen specific CD8 T cells on mesothelin-expressing salivary gland cancer. BMC Cancer. 2018;18:1254.

75. Min IM, Shevlin E, Vedvyas Y, Zaman M, Wyrwas B, Scognamiglio T, et al. CAR T therapy targeting ICAM-1 eliminates advanced human thyroid tumors. Clin Cancer Res. 2017;23:7569-83.

76. Schlumberger M, Tahara M, Wirth LJ, Robinson B, Brose MS, Elisei R, et al. Lenvatinib versus placebo in radioiodine-refractory thyroid cancer. N Engl J Med. 2015;372:621-30.

77. Brose MS, Robinson B, Sherman SI, Jarzab B, Lin CC, Vaisman F, et al. Cabozantinib versus placebo in patients with radioiodine-refractory differentiated thyroid cancer who have progressed after prior VEGFR-targeted therapy: Results from the phase 3 COSMIC-311 trial. 2021;39:6001.

78. Filetti S, Durante C, Hartl D, Leboulleux S, Locati LD, Newbold K, et al; ESMO Guidelines Committee. Thyroid cancer: ESMO clinical practice guidelines for diagnosis, treatment and follow-up. Ann Oncol. 2019;30:1856-83.

79. Schlumberger M, Elisei R, Müller S, Schöffski P, Brose M, Shah M, et al. Overall survival analysis of EXAM, a phase III trial of cabozantinib in patients with radiographically progressive medullary thyroid carcinoma. Ann Oncol. 2017;28:2813-19.

80. Subbiah V, Kreitman RJ, Wainberg ZA, Cho JY, Schellens JHM, Soria JC, et al. Dabrafenib and trametinib treatment in patients with locally advanced or metastatic BRAF V600-mutant anaplastic thyroid cancer. J Clin Oncol. 2018;36:7-13.

81. Mehnert JM, Varga A, Brose MS, Aggarwal RR, Lin CC, Prawira A, et al. Safety and antitumor activity of the anti-PD-1 antibody pembrolizumab in patients with advanced, PD-L1-positive papillary or follicular thyroid cancer. BMC Cancer. 2019;19:196.

82. Takahashi S, Kiyota N, Yamazaki T, Chayahara N, Nakano K, Inagaki L, et al. A phase II study of the safety and efficacy of lenvatinib in patients with advanced thyroid cancer. Future Oncol. 2019;15:717-26.

83. Haugen B, French J, Worden FP, Konda B, Sherman EJ, Dadu R, et al. Lenvatinib plus pembrolizumab combination therapy in patients with radioiodine-refractory (RAIR), progressive differentiated thyroid cancer (DTC): results of a multicenter phase II international thyroid oncology group trial. J Clin Oncol. 2020;38:6512.

84. Haugen B, French JD, Worden F, Konda B, Sherman EJ, Dadu R, et al. 1917P pembrolizumab salvage add-on therapy in patients with radioiodine-refractory (RAIR), progressive differentiated thyroid cancer (DTC) progressing on lenvatinib: results of a multicenter phase II International Thyroid Oncology Group Trial. Ann Oncol. 2020;31:S1086.

85. Lorch JH, Barletta JA, Nehs M, Uppaluri R, Alexander EK, Haddad RI, et al. A phase II study of nivolumab (N) plus ipilimumab (I) in radioidine refractory differentiated thyroid cancer (RAIR DTC) 
with exploratory cohorts in anaplastic (ATC) and medullary thyroid cancer (MTC). J Clin Oncol. 2020;38:6513.

86. Wirth LJ, Eigendorff E, Capdevila J, Paz-Ares LG, Lin CC, Taylor MH, et al. Phase I/II study of spartalizumab (PDR001), an anti-PD1 mAb, in patients with anaplastic thyroid cancer. J Clin Oncol. 2018;36:6024.

87. Capdevila J, Wirth LJ, Ernst T, Ponce Aix S, Lin CC, Ramlau R, et al. PD-1 blockade in anaplastic thyroid carcinoma. J Clin Oncol. 2020;38:2620-7.

88. Cabanillas ME, Dadu R, Ferrarotto R, Liu S, Fellman BM, Gross ND, et al. Atezolizumab combinations with targeted therapy for anaplastic thyroid carcinoma (ATC). J Clin Oncol. 2020;38:6514.

89. French JD. Immunotherapy for advanced thyroid cancers-rationale, current advances and future strategies. Nat Rev Endocrinol. 2020;16:629-41.

90. Leboulleux S, Godbert Y, Penel N, Hescot S, de la Fouchardiere C, Blonski M, et al. Benefits of pembrolizumab in progressive radioactive iodine refractory thyroid cancer: results of the AcSé pembrolizumab study from unicancer. J Clin Oncol. 2021;39:6082.

91. El-Naggar AK, Chan JKC, Grandis JR, Takata T, Slootweg PJ. WHO classification of head and neck tumours. 4th ed. France: IARC; 2017.

92. Girelli L, Locati L, Galeone C, Scanagatta P, Duranti L, Licitra L, et al. Lung metastasectomy in adenoid cystic cancer: Is it worth it? Oral Oncol. 2017;65:114-8.

93. Bergamini C, Cavalieri S, Cascella T, Lanocita R, Alfieri S, Resteghini C, et al. Local therapies for liver metastases of rare head and neck cancers: a monoinstitutional case series. Tumori. 2021;107:188-95.

94. Tchekmedyian V, Sherman EJ, Dunn L, Tran C, Baxi S, Katabi N, et al. Phase II study of lenvatinib in patients with progressive, recurrent or metastatic adenoid cystic carcinoma. J Clin Oncol. 2019;37:1529-37.

95. Locati LD, Galbiati D, Calareso G, Alfieri S, Singer S, Cavalieri S, et al. Patients with adenoid cystic carcinomas of the salivary glands treated with lenvatinib: activity and quality of life. Cancer. 2020;126:1888-94.

96. Cavalieri S, Platini F, Bergamini C, Resteghini C, Galbiati D, Bossi P, et al. Genomics in non-adenoid cystic group of salivary gland cancers: one or more druggable entities? Expert Opin Investig Drugs. 2019;28:435-43.

97. Locati LD, Perrone F, Cortelazzi B, Lo Vullo S, Bossi P, Dagrada G, et al. Clinical activity of androgen deprivation therapy in patients with metastatic/relapsed androgen receptor-positive salivary gland cancers. Head Neck. 2016;38:724-31.

98. Takahashi H, Tada Y, Saotome T, Akazawa K, Ojiri H, Fushimi C, et al. Phase II trial of trastuzumab and docetaxel in patients with human epidermal growth factor receptor 2-positive salivary duct carcinoma. J Clin Oncol. 2019;37:125-34.

99. Bando H, Kinoshita I, Modi S, Tsurutani J, Bang YJ, Iwata H, et al. Trastuzumab deruxtecan (T-DXd) in patients with human epidermal growth factor receptor 2 (HER2)-expressing salivary duct carcinoma: subgroup analysis of two phase 1 studies. J Clin Oncol. 2021;39:6079.

100. Linxweiler M, Kuo F, Katabi N, Lee M, Nadeem Z, Dalin MG, et al. The immune microenvironment and neoantigen landscape of aggressive salivary gland carcinomas differ by subtype. Clin Cancer Res. 2020;26:2859-70.

101. Ho AS, Ochoa A, Jayakumaran G, Zehir A, Valero Mayor C, Tepe J, et al. Genetic hallmarks of recurrent/ metastatic adenoid cystic carcinoma. J Clin Invest. 2019;129:4276-89.

102. Cohen RB, Delord JP, Doi T, Piha-Paul SA, Liu SV, Gilbert J, et al. Pembrolizumab for the treatment of advanced salivary gland carcinoma: findings of the phase 1b KEYNOTE-028 study. Am J Clin Oncol. 2018;41:1083-8.

103. Niwa K, Kawakita D, Nagao T, Takahashi H, Saotome T, Okazaki M, et al. Multicentre, retrospective study of the efficacy and safety of nivolumab for recurrent and metastatic salivary gland carcinoma. Sci Rep. 2020;10:16988. 
104. Burman B, Sherman EJ, Dunn L, Fetten JV, Michel LS, Morris LGT, et al. A phase II trial cohort of nivolumab plus ipilimumab in patients (pts) with recurrent/metastatic salivary gland cancers (R/M SGCs). 2021;39:6002.

105. Chen YP, Chan ATC, Le QT, Blanchard P, Sun Y, Ma J. Nasopharyngeal carcinoma. Lancet. 2019;394:64-80.

106. Bossi P, Chan AT, Licitra L, Trama A, Orlandi E, Hui EP, et al; ESMO Guidelines Committee. Nasopharyngeal carcinoma: ESMO-EURACAN clinical practice guidelines for diagnosis, treatment and follow-up. Ann Oncol. 2021;32:452-65.

107. Zhang L, Huang Y, Hong S, Yang Y, Yu G, Jia J, et al. Gemcitabine plus cisplatin versus fluorouracil plus cisplatin in recurrent or metastatic nasopharyngeal carcinoma: a multicentre, randomised, open-label, phase 3 trial. Lancet. 2016;388:1883-92.

108. Park JC, Wirth LJ, Clark JR, Durbeck J, Ho WJ, Boudadi K, et al. Immune checkpoint inhibitor in nasopharyngeal carcinoma: multi-institution experience. J Clin Oncol. 2020;38:6538.

109. Fang W, Zhang J, Hong S, Zhan J, Chen N, Qin T, et al. EBV-driven LMP1 and IFN- $\gamma$ up-regulate PD-L1 in nasopharyngeal carcinoma: Implications for oncotargeted therapy. Oncotarget. 2014;5:12189-202.

110. Jain A, Chia WK, Toh HC. Immunotherapy for nasopharyngeal cancer-a review. Chin Clin Oncol. 2016;5:22.

111. Ma BBY, Lim WT, Goh BC, Hui EP, Lo KW, Pettinger A, et al. Antitumor activity of nivolumab in recurrent and metastatic nasopharyngeal carcinoma: an international, multicenter study of the mayo clinic phase 2 consortium (NCI-9742). J Clin Oncol. 2018;36:1412-8.

112. Fang W, Yang Y, Ma Y, Hong S, Lin L, He X, et al. Camrelizumab (SHR-1210) alone or in combination with gemcitabine plus cisplatin for nasopharyngeal carcinoma: results from two single-arm, phase 1 trials. Lancet Oncol. 2018;19:1338-50.

113. Zhang L, Yang Y, Chen X, Li J, Pan J, He X, et al. 912MO a single-arm, open-label, multicenter phase II study of camrelizumab in patients with recurrent or metastatic (R/M) nasopharyngeal carcinoma (NPC) who had progressed on $\geq 2$ lines of chemotherapy: CAPTAIN study. Ann Oncol. 2020;31:S659.

114. Nivolumab in treating patients with recurrent and/or metastatic nasopharyngeal cancer [Internet]. USA: National Library of Medicine; c2021 [cited 2021 Apr 1]. Available from: https://clinicaltrials.gov/ ct2/show/NCT02339558

115. Yang Y, Qu S, Li J, Hu C, Xu M, Li W, et al. Camrelizumab versus placebo in combination with gemcitabine and cisplatin as first-line treatment for recurrent or metastatic nasopharyngeal carcinoma (CAPTAIN-1st): a multicentre, randomised, double-blind, phase 3 trial. Lancet Oncol. 2021;22:1162-74.

116. Amit M, Abdelmeguid AS, Watcherporn T, Takahashi H, Tam S, Bell D, et al. Induction chemotherapy response as a guide for treatment optimization in sinonasal undifferentiated carcinoma. J Clin Oncol. 2019;37:504-12.

117. Orlandi E, Cavalieri S, Granata R, Nicolai P, Castelnuovo P, Piazza C, et al. Locally advanced epithelial sinonasal tumors: the impact of multimodal approach. Laryngoscope. 2020;130:857-65.

118. De Cecco L, Serafini MS, Facco C, Granata R, Orlandi E, Fallai C, et al. A functional gene expression analysis in epithelial sinonasal cancer: biology and clinical relevance behind three histological subtypes. Oral Oncol. 2019;90:94-101.

119. Stelow EB, Bellizzi AM, Taneja K, Mills SE, Legallo RD, Kutok JL, et al. NUT rearrangement in undifferentiated carcinomas of the upper aerodigestive tract. Am J Surg Pathol. 2008;32:828-34.

120. Evans AG, French CA, Cameron MJ, Fletcher CD, Jackman DM, Lathan CS, et al. Pathologic characteristics of NUT midline carcinoma arising in the mediastinum. Am J Surg Pathol. 2012;36:1222-7.

121. Gelsomino F, Lamberti G, Tiseo M, Rocco D, Pasello G, Cecere FL, et al. Atezolizumab in a CoHort of pretreated, advanced, non-small cell lung cancer patients with rare histological subtypes (CHANCE trial). Ther Adv Med Oncol. 2020;12:1758835920915983. 